\title{
A descriptive analysis of reasons for early childhood caries among a selected group of Sri Lankan preschool children
}

Chathurika Dissanayaka ${ }^{1}, *$ Manori Gamage $^{2}$

Sri Lanka Journal of Child Health, 2020; 49(3): 246-250

\begin{abstract}
Introduction: Dental caries is a microbial disease of the calcified tissues of the teeth. When a child has one or more decayed missing (due to caries) or filled tooth surfaces (DMFT) in any primary teeth by 71 months or younger it is defined as early childhood caries (ECC).
\end{abstract}

Objectives: To analyse the dietary habits and oral hygienic practices among a selected group of preschool children who were diagnosed to suffer from ECC, using their primary caregiver responses.

Method: A cross sectional study was conducted among primary caregivers of children 1-5 years old with proven dental caries who were referred to the Dental Institute Maharamaga from September to December 2018. Primary caregivers of mentally retarded children and caregivers who were not familiar with the child's routine were excluded.

Results: Total number of primary caregivers involved in the study was 213. Among them 194 (91\%) were mothers. Among the children included in the study, $104(51.2 \%)$ were girls. The children had a mean age of 42 months with the majority belonging to the 49-60 month age group. Majority $(60 \%)$ of children were introduced to use a toothbrush between 1-2 years of age and only 2 $(0.9 \%)$ started to use a toothbrush after 2 years of age. The majority $(86.9 \%)$ of caregivers stated that their children do tooth brushing twice a day and $85.9 \%$ stated that they themselves brush their

${ }^{1}$ BSc Nursing Undergraduate, Department of Allied
Health Sciences, University of Sri
Jayewardenepura, ${ }^{2}$ Senior Lecturer, Faculty of
Medical Sciences, University of Sri
Jayewardenepura, Sri Lanka and Honorary
Consultant Paediatrician, Colombo Teaching
Hospital, Sri Lanka
*Correspondence: manorigamage@sjp.ac.lk
iD orcid.org/0000-0003-3836-5945

(Received on 26 October 2019: Accepted after revision on 20 December 2019)

The authors declare that there are no conflicts of interest

Personal funding was used for the project.

Open Access Article published under the Creative

Commons Attribution CC-BY (c) (P) License child's teeth. The majority $(98.1 \%)$ of caregivers stated that they use toothpaste to brush their child's teeth and $88 \%$ stated that the toothpaste they use contains fluoride. Ninety seven $(45.5 \%)$ caregivers were still breast feeding at the time of the study and of them 41(42.3\%) breast fed during sleep. Only 52 $(24.4 \%)$ children were bottle fed. Of the carers of bottle-fed children, $56 \%$ stated that their children bottle-fed during bedtime. Thirty two (61.5\%) caregivers of bottle-fed children used to add sugar to child's milk bottle. Furthermore, 32 (61.5\%) caregivers had not practised mouth washing after bottle-feeding.

http://dx.doi.org/10.4038/sljch.v49i3.9142

Conclusions: In this study 213 children had proven ECC despite the majority of caregivers introducing their children to the tooth brush between 1-2 years of age, themselves brushing their children's teeth twice/day and using toothpaste containing fluoride. Almost all children consumed sugary food items, such as biscuits and sugar containing milk products more than once daily. Two other possible reasons for the high rate of ECC in this study were not washing the children's mouths after they consumed sugary food and breast feeding during sleep.

(Key words: Early childhood caries, ECC, oral hygiene, dietary habits, DMFT score, breastfeeding)

\section{Introduction}

Dental caries is a problem in children and adults ${ }^{1}$. It is described as a microbial disease of the calcified tissues of the teeth with demineralization of the inorganic portion, and destruction of the organic substance of the tooth ${ }^{2}$. When a child has one or more decayed, missing (due to caries), or filled tooth surfaces (DMFT) in any primary teeth by 71 months, it is defined as early childhood caries $(\mathrm{ECC})^{3}$. Severity of ECC is assessed using DMFT values $^{3}$. Identified risk factors for dental caries include past and current dental status, current medical status, tooth location, life style, diet and dietary habits, oral hygiene, oral bio film formation and lack of fluoride 4 . Remaining food particles and nutrients through saliva, makes the oral cavity ideal for microorganisms of which Streptococcus mutans is a well-recognized organism metabolising sugary food into acids leading to demineralisation of the enamel of the teeth ${ }^{5}$. 
In most developed countries, ECC prevalence is from $1 \%$ to $12 \%$ but in developing countries it is as high as $70 \%{ }^{6}$. A Sri Lankan study in 2003 reported that the ECC prevalence in preschool children was $23 \%{ }^{7}$. However, according to the National Oral Health Survey Sri Lanka 2015-2016, ECC prevalence among children less than 5 years was $63 \%{ }^{8}$. Major factors contributing to the high prevalence of ECC in developing countries include poor socioeconomic status, which is closely linked with poor parental education, poor hygienic practices and unhealthy dietary habits and limited availability of dental services ${ }^{9}$. Although many worldwide studies have shown that dietary habits and poor oral hygienic practices are the most important risk factors for ECC, very few Sri Lankan studies have looked into the association between dietary habits and hygienic practices in children with dental caries.

\section{Objectives}

To analyse the dietary habits and oral hygienic practices among a selected group of preschool children who were diagnosed to suffer from ECC, using their primary caregiver responses.

\section{Method}

A descriptive cross sectional study was conducted after obtaining ethical clearance from the Ethics Review Committee of the Faculty of Medical Sciences, University of Sri Jayewardenepura.
Primary caregivers of children, aged 1-5 years with proven dental caries, who presented to the Dental Institute Maharamaga from September to December 2018, were included in the study after written informed consent from the caregiver. Primary caregivers of mentally retarded children and caregivers who were not familiar with the child's routine were excluded. Data was collected using a pre-tested self-administered questionnaire. Demographic data, data related to dietary habits and data on oral hygienic practices were collected from primary caregivers.

\section{Results}

The total number of primary caregivers involved in the study was 213 of whom 194 (91\%) were mothers. Of the $213,57.7 \%$ were educated up to GCE ordinary level. All fathers were working while $77 \%$ of mothers were housewives. Among the children included, 104 (51.2\%) were girls. Majority of children belonged to the age group 4960 months with a mean age of 42 months.

Firstly, the oral hygienic practices related to tooth brushing were assessed. All 213 children did brush their teeth routinely, $60 \%$ were introduced to use a toothbrush between 1-2 years of age, 98\% used tooth paste to brush their child's teeth and $87 \%$ stated that their children did tooth brushing twice a day (Table 1).

Table 1: Oral hygienic practices related to tooth brushing $(n=213)$

\begin{tabular}{|l|c|}
\hline \multicolumn{1}{|c|}{ Characteristic } & Frequency (\%) \\
\hline Age at which the child started to use a tooth brush & \\
Before 1 year & $84(39.4)$ \\
Between 1-2 years & $127(59.6)$ \\
After 2 years & $02(0.9)$ \\
\hline Usage of tooth paste & $209(98.1)$ \\
Yes & $04(01.9)$ \\
No & $19(08.9)$ \\
\hline Frequency of tooth brushing & $185(86.9)$ \\
Once a day & $09(04.2)$ \\
Twice a day & \\
More than twice a day & \\
\hline
\end{tabular}

Of the primary caregivers $183(85.9 \%)$ stated that they themselves brush their child's teeth, whilst 8 $(3.8 \%)$ stated that their child brushes teeth solely by himself or herself. Whilst $184(88 \%)$ caregivers stated that the toothpaste they used contained fluoride, the rest did not know whether it had fluoride or not. None of the primary caregivers had ever used mouthwashes for their children.

Next, we analysed the food habits and practices of these children and found that $97(45.5 \%)$ were still being breast fed, $42.3 \%$ of the 97 feeding from the breast during sleep (Table 2). Of the 116 not currently breastfeeding, $36.2 \%$ had terminated breast-feeding after 2 years of age. Of the 213 children only $52(24.4 \%)$ were bottle fed (Table 2$)$. Of the 29 , three $(10.3 \%)$ caregivers mentioned that their children sleep while feeding from the bottle.

Whilst $44(84.6 \%)$ caregivers used the feeding bottle only to give milk, the rest used it also to give juices and water. Of the 52 caregivers involved in bottle feeding, $32(61.5 \%)$ added a teaspoonful or less of sugar to the child's feeding bottle. Of the 52 caregivers, $32(61.5 \%)$ did not wash their child's mouth after bottle-feeding. 
Table 2: Practices related to breast and bottle feeding $(n=213)$

\begin{tabular}{|l|c|}
\hline \multicolumn{1}{|c|}{ Practice } & Frequency (\%) \\
\hline Breast fed at the time of the study & $97(45.5)$ \\
Yes & $116(54.5)$ \\
No & \\
\hline Of the breast feeders, breast fed during sleep & $41(42.3)$ \\
Yes & $56(57.7)$ \\
No & \\
\hline Bottle fed at the time of the study & $52(24.4)$ \\
Yes & $161(75.6)$ \\
No & \\
\hline Bottle fed at night & $29(56.0)$ \\
Yes & $23(44.0)$ \\
No & \\
\hline
\end{tabular}

In our study, almost all children consumed sugary food items more than once daily and of them the commonest was biscuits $(54.5 \%)$, which was only second to sugar containing milk products $(38 \%)$. It was noted that $68.1 \%$ of the caregivers did not wash their child's mouth after consuming sugary food and the rest mentioned that they practised it occasionally. When asked about usage of routine dental care facilities for their children, none had routine planned visits before their children developed dental caries and $89 \%$ of them responded that they did not know that routine visits were recommended when children were not suffering from any dental issues.

\section{Discussion}

Dental caries among children is a well-known factor for school absenteeism ${ }^{10}$. In one study it was shown that dental caries may affect the weight gain in children by causing pain and discomfort, sleeping disturbance affecting psychological wellbeing and interference with feeding ${ }^{11}$. Studies have found that increased consumption of sugary food, the prolonged period of time sugary foods remain on teeth, unhealthy dietary habits, poor oral hygienic practices, and poor usage of dental care facilities, are major factors responsible for $\mathrm{ECC}^{12}$. A study in Abu Dhabi concluded that consumption of high sugar containing food and infrequent tooth brushing have a strong association with $\mathrm{ECC}^{13}$. A study by Elidrissi et al found that children who initiated tooth brushing at an earlier age and who brushed teeth three times a day have very significantly low DMFT scores ${ }^{14}$. An Indian study in 2015 found that consumption of snacks three or more times, sticky foods and syrup medications increased the prevalence of $\mathrm{ECC}^{15}$. On the other hand, a study by Ngatia et al concluded that dental caries risk at 30 months of age was more with exclusively or partially breast fed babies than exclusively formula fed babies ${ }^{16}$. In this study prevalence of dental caries was higher among males than females ${ }^{16}$. In our study population $51 \%$ were female. Most children in our study belonged to the 4-5 year age category. This was supported by a study in Sudan which concluded that dental caries increased with age, the highest prevalence being in the 3-5 year age group ${ }^{14}$. Most likely, children in this age category have more access to sugary and sticky food than younger children.

Regular and frequent tooth brushing is known to reduce $\mathrm{ECC}^{14}$. Early introduction of tooth brush to children, twice a day or more tooth brushing, and usage of a fluoride containing tooth paste are also known to reduce the prevalence $\mathrm{ECC}^{17}$. In our study population, all children did tooth brushing as an oral hygienic practice, and in $86 \%$, brushing was done by an adult. Around $60 \%$ children were introduced to the tooth brush between 1-2 years of age. Furthermore, $98 \%$ of caregivers used toothpaste to clean their child's teeth, $88 \%$ knew that they used a fluoride containing tooth paste and $87 \%$ brushed their children's teeth twice a day. Despite all the above measures which should have reduced the incidence of ECC, all 213 study participants had proven ECC needing referral to this tertiary dental care institution. This clearly indicates that factors other than poor tooth brushing contributed to the development ECC in this selected group of preschool children. This is supported by a Sri Lankan study by Kumarihamy et al in 2011 in a group of preschool children from selected $\mathrm{MOH}$ areas of Colombo, which showed a significant relationship between intake of milk with sugar, sweet consumption and $\mathrm{ECC}^{18}$. Let us consider some of these other factors which may have contributed to the ECC in our study.

On analysing the dietary habits of our study population, there were dietary habits and practices among them which are known to increase the prevalence of ECC. These include increased and frequent consumption of sugary food and not cleaning mouth after such consumption, breast and bottle feeding while sleeping and not practising mouth washing following bedtime milk feeds before sleep. 
Almost all our study subjects, consumed sugary food items, such as biscuits $(54.5 \%)$ and sugar containing milk products (38\%) more than once daily. Further, $68 \%$ of the caregivers did not wash their children's mouths after they had consumed sugary food. Of these preschool children, 97 $(45.5 \%)$ were still being breast fed while 52 (24.4\%) were being bottle fed. Of the 97 currently being breast fed, $41(42.3 \%)$ continued to be breast fed during sleep. Of the 52 being bottle fed, 29 $(55.8 \%)$ continued to be bottle fed during bedtime and 3 children used to sleep while being bottle fed. Further, $32(61.5 \%)$ of the bottle fed babies got sugar added to their feeds by their primary caregivers, who furthermore. did not brush their teeth or wash their children's mouths after the bedtime feed. Thus, it is clearly shown that these dietary practices and habits made these preschool children develop significant dental caries which even needed a tertiary care referral.

To prevent ECC, parental influence is very important from early infant feeding, including breast feeding practices up to proper usage of dental facilities and establishing good oral hygienic practices. Mothers play a major role in establishing healthy dietary habits, food selection and dental hygienic practices. Unfortunately, there is no properly implemented primary preventive programmes for dental care in Sri Lanka. Moreover, even available facilities are limited to a few centres which have prolonged waiting times to get an appointment. This may be the reason why the majority of primary caregivers did not know about or attend preventive regular checkups. ECC is a preventable problem among preschool children, which is strongly associated with dietary and oral hygienic practices. It was noted that the major reason why they got it was due to dietary habits and practices, which could be easily, corrected with health education practices. In addition, it is strongly recommended to establish a proper primary preventive dental practices which are readily available and accessible to primary care takers.

\section{Conclusions}

In this study at the Dental Institute Maharamaga, 213 children had proven ECC despite the majority of caregivers introducing their children to the tooth brush between 1-2 years of age, themselves brushing their children's teeth twice a day and using toothpaste containing fluoride. Almost all children consumed sugary food items, such as biscuits and sugar containing milk products more than once daily. Two other possible reasons for the high rate of ECC in this study were not washing the children's mouths after they consumed sugary food, and breast feeding during sleep.

\section{References}

1. Peterson PE. The World Oral Health Report 2003: continuous improvement of oral health, in the $21^{\text {st }}$ century - the approach of the WHO Global Oral Health Programme. Community Dentistry and Oral Epidemiology 2003; 31 Suppl1:3-23.

2. Rajendran A, Sivapathasundharam B, editors. Shafer's Textbook of Oral Pathology. $6^{\text {th }}$ ed. Elsevier India 2009.

3. De Drauwe A, Aps JK, Martens LC. Early childhood caries (ECC): What's in a name? European Journal of Paediatric Dentistry 2004; 5(2) 62-70.

4. Islam B, Khan SN, Khan AU. Dental carries: from infection to prevention. Medical Science Monitor 2007; 13(11): RA 196-203.

5. Vadiakas G. Case definition, aetiology and risk assessment of early childhood caries (ECC): a revisited review. European Archives of Paediatric Dentistry 2008; 9(3):114-25.

6. Congiu G, Campus G, Luglie PF. Early childhood caries (ECC) prevalence and background factors: A review. Oral Health and Preventive Dentistry 2014; 12(1): 71-6.

7. Shahim FN. Factors of risk of early childhood caries in a selected district in Sri Lanka, Post Graduate Institute of Medicine, Colombo PGIM; NSF. 2003.

8. National Oral Health Survey Sri Lanka. 2015-2016. Ministry of Health, Nutrition and Indigenous Medicine December 2018.

9. Amin TT, Al-Abad BM. Oral hygiene practices, dental knowledge, dietary habits and their relation to caries among male primary school children in Al Hassa, Saudi Arabia. International Journal of Dental Hygiene 2008; 6(4):361-70.

10. Jackson SL, Vann WF Jr, Kotch JB, Pahel BT, Lee JY. Impact of poor oral health on children's school attendance and performance. American Journal of Public Health 2011; 101(10):1900-6. 
11. Sheiham A. Dental caries affects body weight, growth and quality of life in preschool children. British Dent Journal 2006; 201(10):625-6.

12. Kowash MB. Severity of early childhood caries in preschool children attending AlAin Dental Centre, United Arab Emirates. European Archives of Paediatric Dentistry 2015; 16(4):319-24.

13. Elamin A, Garemo M, Gardner A. Dental caries and their association with socioeconomic characteristics, oral hygiene practices and eating habits among preschool children in Abu Dhabi, United Arab Emirates - the NOPLAS project. BMC Oral Health 2018; 18:104.

14. Elidrissi SM, Naidoo S. Prevalence of dental caries and tooth brushing habits among preschool children in Khartoum State, Sudan. International Dental Journal 2016; 66(4):215-20.

15. Kuriakose S, Prasannan M, Remya KC, Kurian J, Sreejith KR. Prevalence of early childhood caries among preschool children in Trivandrum and its association with various risk factors Contemporary Clinical Dentistry 2015; 6(1):69-73.
16. Ngatia EM, Imungi JK, Muita JW, Nganga PM. Dietary patterns and dental caries in nursery school children in Nairobi, Kenya. East African Medical Journal 2001; 78(12):673-7.

17. Moimaz SAS, Martins RJ, Forte FDS, Saliba NA. () Oral hygiene practices, parents' education level and dental caries pattern in 0 to 5 years-old children. Brazilian Journal of Oral Sciences 2015; 4(14): 778-82.

18. Kumarihamy SL, Subasinghe LD, Jayasekara P, Kularatna SM, Palipana PD. The prevalence of early childhood caries in 1-2 yrs olds in a semi-urban area of Sri Lanka. BMC Research Notes 2011; 4:336. 